\title{
Modifications in a Reference Freeze-Dried Direct Agglutination Test to Improve Visceral Leishmaniasis Detection
}

\author{
Abdallah el Harith, ${ }^{1 \star}$ Yousif Awad, ${ }^{1}$ Abdelhafeiz Mahamoud, ${ }^{1}$ Elfadil Abass, ${ }^{2}$ Durria Mansour, ${ }^{3}$ Claudia Moura de Melo, ${ }^{4}$ \\ Rubens Riscala Madi, ${ }^{4}$ Saul J. Semiao-Santos, ${ }^{5}$ and Hussam Ali Osman ${ }^{1}$ \\ ${ }^{1}$ School of Pharmacy, Ahfad University for Women, Omdurman, Sudan; ${ }^{2}$ Department of Clinical Laboratory Science, College of Applied Medical \\ Sciences, Imam Abdulrahman Bin Faisal University, Dammam, Saudi Arabia; ${ }^{3}$ School of Health Sciences, Ahfad University for Women, Omdurman, \\ Sudan; ${ }^{4}$ Program in Health and Environment, University of Tiradentes, Aracaju, Brazil; ${ }^{5}$ Department of Medicine and Nursing, University of
} Tiradentes, Aracaju, Brazil

\begin{abstract}
Currently, a significantly lower temperature $\left(35^{\circ} \mathrm{C}\right)$ than initially established $\left(56^{\circ} \mathrm{C}\right)$ is indicated as the maximum temperature storage for the commercial reference visceral leishmaniasis (VL) freeze-dried direct agglutination test (FD-DAT). Despite an approximately $50 \%$ loss in the number of promastigotes in an FD-DAT batch that expired 7 years earlier, the promastigotes maintained a similar morphology to the equivalent valid batch implying most likely that auto-agglutination, rather than aging, is the main reason for expiry. The substitution of normal saline which was initially recommended for reconstitution, by citrate-saline/formaldehyde (CSF) as an anti-clumping/preservative agent resulted in restoration of validity comparable with that of the freeze-dried original or the liquid direct agglutination test (LQ-DAT) version (Friedman ANOVA test $=1.0588 ; P=0.5890$ ). Following a similar reconstitution procedure as for the 7-year expired antigen, using significantly lower promastigote concentration $\left(1.4 \times 10^{7} / \mathrm{mL}\right)$ than in the non-expired $\left(9.0 \times 10^{7} / \mathrm{mL}\right)$, good reliability for VL detection and stability at $4^{\circ} \mathrm{C}(>12$ months) were achieved. In comparison with the original version using normal saline (\$32.0/vial), the cost-effectiveness of the FD-DAT was appreciably improved by the CSF incorporation and lowering of promastigote concentration per unit suspension medium $(\$ 12.8 / \mathrm{vial})$. With diagnostic reliability comparable with the full-out titration used, FD-DAT procedure based on single sample dilution at the VL cutoff $(1: 3,200)$ permitted the use of significantly smaller antigen volumes $(0.1 \mathrm{~mL}$ vs. $>1.5 \mathrm{~mL})$, therefore contributing to a further reduction in the application cost. The successful replacement of $\beta$-mercaptoethanol $(\beta-\mathrm{ME})$ by urea $(T=21.00 ; P=0.0868)$ provided the required safety for the test procedure similar to the widely applied LQ-DAT.
\end{abstract}

\section{INTRODUCTION}

To date, the direct agglutination (DAT) and rK39 rapid tests are generally accepted as being the most reliable tests for visceral leishmaniasis (VL) detection worldwide. ${ }^{1}$ However, in the course of laboratory and field application during the past 25-28 years in Sudan, both tests revealed several drawbacks that negatively impacted their application on a routine basis. $^{2-5}$ The limited shelf-life ( \pm 30 days) of the rK39 at ambient temperatures and high application cost of the freezedried direct agglutination test (FD-DAT) $( \pm \$ 32.0$ /vial vs average income in Sudan of $\$ 70.0$ a month) were the most important shortcomings. ${ }^{2}$ The strict temperature range for the test kit $\left(5-35^{\circ} \mathrm{C}\right)$ limited the use of the FD-DAT in Sudan, particularly during the hot season (April-October) when the mid-day temperatures of $\pm 40^{\circ} \mathrm{C}$ are considered normal almost throughout the whole country. Equally disadvantageous is the short validity of the antigen after reconstitution ( $\leq 48$ hours) in normal saline as instructed ( $5-\mathrm{mL}$ vials, enough for testing eight patients). If the remaining reconstituted portion (enough for testing seven patients) is not used within this short period, it will no longer be valid for use because of autoagglutination. The obligatory use of the rather expensive fetal calf serum $( \pm \$ 400 / L)$ in the test procedure has also increased he costs of the kit production. The exclusive use of Leishmania donovani (strain MHOM/SD/68/1-S) as a source of the antigen has negatively impacted the sensitivity of the test when compared with the liquid direct agglutination test (LQDAT) equivalent, in which corresponding endemic strains

*Address correspondence to Abdallah el Harith, School of Pharmacy, Ahfad University for Women, P.O. Box 167, Alardah Street, Omdurman, Sudan 14411. E-mail: abdallah.elharith@gmail.com were used instead. ${ }^{2,3}$ The use of $\beta$-mercaptoethanol ( $\beta$-ME) in the FD-DAT procedure has confined the test application to laboratories exclusively equipped with fume chamber facility. The formation of a blue background around the negative dot that signals the end-point of the agglutination reaction (most likely due to freeze-drying) renders determination of the exact titer in the test sample rather difficult. Whether handling steps other than freeze-drying during kit production contribute to stain shedding from Coommassie Brilliant Blue remains unclear. However, we still believe that FD-DAT possess better potential to confirm the results obtained with LQ-DAT because of being more sensitive and semi-quantitative than the rK39 strip test, particularly in the economically less privileged endemic areas of VL in East Africa.

Microscopic examination performed on samples from valid and expired FD-DAT batches, reconstituted as instructed in normal saline, revealed no significant difference in the morphology of the promastigotes. However, despite the difficulty in determining the exact reason(s) for the invalidity of the expired FD-DAT batches, we believe that lowering of repulsion forces between suspended cells leads to an enhanced tendency for aggregation, that is, auto-agglutination.

Because of improvements introduced earlier to the LQ-DAT by using the citrate-saline as the anti-clumping agent, significant extension of the antigen shelf-life (12-72 months) was achieved at $4^{\circ} \mathrm{C}$. $^{6-9}$ To enhance the FD-DAT application and achieve the same properties as LQ-DAT, we, therefore, decided to follow a similar approach. In doing so, we expected to evade the occurrence of auto-agglutination in the "expired" FD-DAT batches and prolong the shelf-life of the reconstituted antigen, and at the same time creating the valid one. To further reduce FD-DAT application cost, lower promastigote concentration per unit of suspension medium was investigated, 
aiming not to compromise the test reliability for VL detection. A single sample dilution of 1:3,200 (cutoff for VL) was investigated successfully earlier with the LQ-DAT, requiring smaller antigen volumes in comparison with the full-out titration. ${ }^{6}$

\section{MATERIALS AND METHODS}

Expired FD-DAT batch. Leish DAT Antigen (Royal Tropical Institute, Amsterdam, Netherlands), lot no. 0905 in 5-mL vials without production date but with November 2011 as the expiration date, was used. Microscopic examination of the antigen samples after reconstitution in saline from two randomly selected vials revealed promastigote morphology and their intense blue coloration comparable with valid FD-DAT batch (lot no. 1602, 2018) used as control. Using Neubauer's counting chamber, remarkably lower promastigote counts of $4.75 \times 10^{7}$ and $4.90 \times 10^{7} / \mathrm{mL}$ were determined in comparison with the valid batch $\left(8.7 \times 10^{7}\right.$ and $\left.9.0 \times 10^{7} / \mathrm{mL}\right)$.

Non-expired (valid) FD-DAT batch. Leish DAT Antigen (Royal Tropical Institute, Amsterdam, Netherlands), lot no. 1602 in 5-mL vials with production date February 2016 and expiration date August 2018, were used. Following the producer's instructions, the vials were stored at $4-5^{\circ} \mathrm{C}$ or at air-conditioned laboratory temperature $\left(23-26^{\circ} \mathrm{C}\right)$ until used. Microscopic examination of the samples taken after reconstitution in normal saline from two randomly selected vials revealed similar promastigote morphology and color intensities as in the expired batch. Using Neubauer's counting chamber, promastigote concentrations $\left(8.7 \times 10^{7}\right.$ and $\left.9.0 \times 10^{7} / \mathrm{mL}\right)$ were significantly higher than in the expired batch.

Liquid direct agglutination test. A locally produced LQDAT batch was used. As previously reported, an $L$. donovani strain isolated from a VL patient from the Gedarif area in Eastern Sudan was used as a source of the antigen. ${ }^{2-4}$ Irrespective of form, the promastigotes retained the Coommassie Brilliant Blue stain despite repeated washing with the citratesaline solution $(0.056 \mathrm{M}$ sodium citrate and $0.15 \mathrm{M}$ sodium chloride). The produced antigen was then preserved in citratesaline solution supplemented with $1.2 \%(\mathrm{w} / \mathrm{v})$ citrate-saline/ formaldehyde (CSF) at a promastigote concentration of $1.6 \times$ $10^{7} / \mathrm{mL}$ to simultaneously preserve promastigote morphology and evade auto-agglutination.

Human blood and serum samples. In total, 47 blood spots collected onto filter paper and 18 serum samples from patients with VL from our serum bank were included. ${ }^{2-4}$ Thirty other blood and serum samples also from our serum bank from suspects with negative lymph node aspirates, negative LQDAT, and FD-DAT results were used as negative controls.

Canine serum samples. A total of 43 serum samples of different breeds and age classes were included: 21 were from Croatia, 10 with Leishmania-positive and 11 with Leishmanianegative lymph node aspirates, provided by Dr. Franjo Martinkovic, faculty of veterinary medicine, University of Zagreb. Twenty-two other serum samples from Sudanese police dogs were provided by Captain Hassan, Forensic Affairs of the Ministry of Interior, Khartoum North. The last mentioned dog group was maintained while not in duty, continuously indoors at the Police Department.

Reviving antigen validity in an expired FD-DAT batch. $A$ vial of FD-DAT batch that expired in November 2011 (7 years earlier) was reconstituted in normal saline and applied to sera from two VL and two non-VL patients, following the manufacturer's instructions for a valid batch. As expected, no discernible promastigote settling at the bottom of the V-shaped well containing the sample diluent only (gelatin + normal saline) was observed after 18 hours of incubation confirming, therefore, the occurrence of auto-agglutination. To revive the validity, CSF (0.056 M sodium citrate and $0.15 \mathrm{M}$ sodium chloride supplemented with $1.2 \% \mathrm{w} / \mathrm{v}$ formaldehyde) was used for the antigen reconstitution, instead of normal saline.

To determine the lowest promastigote concentration that still elicits a valid agglutination reaction, CSF was dispensed in volumes of $5,6,7$, or $8 \mathrm{~mL}$ to attain corresponding promastigote concentration $\left(4.9 \times 10^{7}, 3.8 \times 10^{7}, 2.6 \times 10^{7}\right.$, and $1.5 \times$ $10^{7} / \mathrm{mL}$ suspension medium). A concentration of $1.5 \times 10^{7} / \mathrm{mL}$ was found to be the lowest (results not shown). The performance of the "expired" batch reconstituted in CSF at this concentration $\left(1.5 \times 10^{7}\right.$ promastigote $\left./ \mathrm{mL}\right)$ was used to assess the diagnostic efficiency and stability following storage at $4^{\circ} \mathrm{C}$ for $1,2,7,30,90,180$, and 360 days against standard $\mathrm{VL}$ and non-VL sera.

Economical FD-DAT antigen use. Vials containing valid FD-DAT (batch lot no. 1602) were reconstituted in normal saline (5 mL/vial) as instructed. Other vials from the same batch were reconstituted in CSF instead of normal saline. The two differently reconstituted vials were then shaken gently by hand and allowed to stand for 30 minutes at an air-conditioned laboratory temperature $\left(23-26^{\circ} \mathrm{C}\right)$. Aliquots were tested for validity against standard VL and non-VL sera at intervals of 1 , 2 , and 7 days.

To determine the lowest promastigote concentration that still results in valid reaction, lots of $5,6,7,8,9,10,11,12$, or $13 \mathrm{~mL}$ CSF were used for the antigen reconstitution to establish corresponding concentrations of $9.0 \times 10^{7}, 7.8 \times 10^{7}$, $6.5 \times 10^{7}, 5.4 \times 10^{7}, 4.7 \times 10^{7}, 3.4 \times 10^{7}, 2.3 \times 10^{7}, 1.4 \times 10^{7}$, and $0.8 \times 10^{7}$ promastigotes $/ \mathrm{mL}$, respectively. The reactivity of those aliquots was then tested against standard $\mathrm{VL}$ and non-VL sera. With the exception of the largest CSF volume $(13 \mathrm{~mL})$, all other eight aliquots revealed comparable valid test results. To assess for the diagnostic performance of this CSFmodified FD-DAT version, the lowest eligible promastigote concentration earlier determined $\left(1.4 \times 10^{7} / \mathrm{mL}\right)$ was also used here. A valid freeze-dried antigen reconstituted accordingly with $12 \mathrm{~mL}$ CSF was also used to determine the stability of the antigen following storage at $4^{\circ} \mathrm{C}$ for $1,2,7,30,90,180$, and 360 days using standard VL and non-VL sera.

Freeze-dried direct agglutination test execution based on single sample dilution. A cutoff dilution of 1:3,200 was also used in this study following a procedure reported previously for LQ-DAT. ${ }^{6}$ In the control well containing the test sample, $50 \mu \mathrm{L}$ diluent (gelatin + saline) was added. An equal volume of the antigen reconstituted in CSF $\left(1.4 \times 10^{7}\right.$ promastigotes $/ \mathrm{mL}$ ) was then added into the two wells containing either the sample diluent or the test (serum/blood) sample. Reading the test result was performed in the same way as the full-out titration procedure, that is, by visualizing the promastigote sedimentation pattern other than the blue dot presented at the bottom of the $\mathrm{V}$-shaped well containing the sample diluent only. ${ }^{10}$

Exclusion of $\boldsymbol{\beta}$-ME in FD-DAT execution. Instead of fetal calf serum, gelatin ( $0.2 \% \mathrm{wt} / \mathrm{vol}$.) dissolved in normal saline was used as a diluent for the blood/serum sample. To ensure complete miscibility, the gelatin/saline mixture was heated up 
to $80^{\circ} \mathrm{C}{ }^{4}$ To reduce nonspecific agglutination, the gelatin diluent was cooled down to room temperature and supplemented with urea $(0.3 \% \mathrm{wt} / \mathrm{vol})$ instead of $\beta$-ME $(0.8 \% \mathrm{v} /)^{4}{ }^{4}$ Execution of the test was the same as for LQ-DAT using $\mathrm{V}$-shaped well microtiter plates. Reading of test results was performed after 18 hours of incubation at laboratory temperature $\left(23^{\circ} \mathrm{C}\right.$ or $\left.\geq 40^{\circ} \mathrm{C}\right)$ using the $1: 3,200$ dilution as cutoff titer for VL.

Data analysis. The Friedman ANOVA test was used to compare the efficiency of the "repaired" expired FD-DAT and the urea-improved FD-DAT compared with the original LQDAT and FD-DAT versions. The Wilcoxon matched pair test was applied to compare the efficiency of the urea-improved FD-DAT in relation to LQ-DAT. Pearson's correlation coefficient was calculated to assess the degree of relationship between the promastigote concentration $/ \mathrm{mL}$ antigen suspension and the cost of application per patient. In all tests, the titration values were $\log$-transformed $(\log x+1)$ and applied with a $5 \% \mathrm{Cl}$ using the Statistica 7.1 software (Stat Soft Inc., Tulsa, OK).

Study design. To assess whether the incorporation of CSF in antigen reconstitution evades the occurrence of autoagglutination, a 7-year-old expired FD-DAT batch was used. The performance of the "expired" FD-DAT antigen (repaired with CSF) was assessed against standard serum and blood samples from VL and non-VL patients. The stability of the antigen in the non-expired "valid" FD-DAT batch was also determined as for the repaired "expired" at $4^{\circ} \mathrm{C}$ following storage for up to 360 days.

To effectively reduce the cost for FD-DAT application, efforts were made to lower the promastigote concentration per unit suspension medium without negatively affecting the test reliability for $\mathrm{VL}$ detection. Performance of a valid FD-DAT batch reconstituted with CSF at promastigote concentrations ranging from $9.0 \times 10^{7}$ down to $0.8 \times 10^{7} / \mathrm{mL}$ suspension medium was evaluated by testing them against VL and non-VL serum samples. In both "valid" and repaired "expired" FD-DAT batches, stability of the CSF-reconstituted antigen was determined following storage at $4^{\circ} \mathrm{C}$ for 360 days.

A further reduction in the FD-DAT application cost was achieved by performing the test on a single sample dilution basis, at 1:3,200. To achieve the desired level of safety for both user and environment, $\beta-\mathrm{ME}$ was replaced by urea in the test procedure. The effect of this improvement was evaluated on the current freeze-dried and liquid versions involving the use of this toxicant.

\section{TABLE 1}

Performance of an "repaired" 7-year expired FD-DAT in comparison with non-expired freeze-dried (FD-DAT and liquid direct agglutination test (LQ-DAT) versions for the detection of VL

\begin{tabular}{|c|c|}
\hline DAT version* & $\begin{array}{l}\text { Number of serum with DAT titer: } \\
<1: 4001: 8001: 1,6001: 3,200 \dagger \\
1: 6,4001: 12,800 \geq 1 ; 25,600\end{array}$ \\
\hline $\begin{array}{l}\text { Repaired “expired” FD-DAT } \\
\text { Non-expired FD-DAT } \\
\text { LQ-DAT }\end{array}$ & 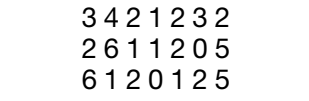 \\
\hline \multicolumn{2}{|c|}{$\begin{array}{l}\text { DAT = direct agglutination; FD-DAT = freeze-dried direct agglutination test; } \mathrm{VL}=\text { visceral } \\
\text { leishmaniasis. } \\
{ }^{\star} \text { No statistical difference between outcomes of the three DAT versions was found (Fr = } \\
\text { 1.0588; } P=0.5890) \text {. } \\
\text { † Cutoff titer for VL. } \\
\text { ‡A 7-year expired FD-DAT batch repaired by substituting normal saline with citrate-saline/ } \\
\text { formaldehyde as anti-clumping/preservative solution. }\end{array}$} \\
\hline
\end{tabular}

\section{RESULTS}

Validity of the 7-year expired FD-DAT batch was successfully restored through the incorporation of CSF as anticlumping/preservative agent in the antigen diluent (Table 1). Except the single aliquot with $0.8 \times 10^{7} / \mathrm{mL}$, all other eight aliquots from the valid FD-DAT batch diluted with CSF at $9.0 \times$ $10^{7}$ down to $1.4 \times 10^{7}$ promastigotes $/ \mathrm{mL}$ demonstrated equally valid and readable agglutination reactions (Figure 1). Therefore, by using this lowest eligible concentration $(1.4 \times$ $10^{7} / \mathrm{mL}$ ) instead of the one originally instructed for FD-DAT

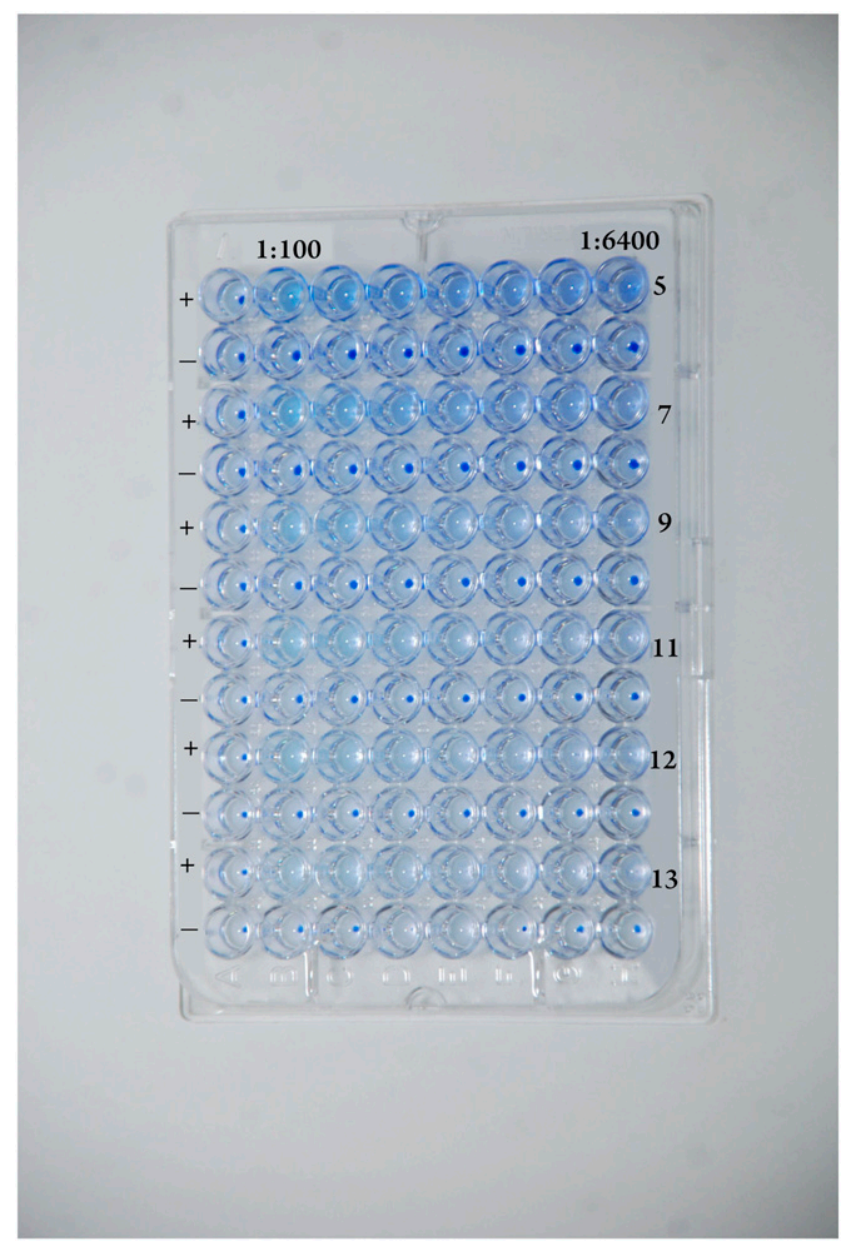

FIGURE 1. An improved procedure for execution of a reference freeze-dried direct agglutination test (FD-DAT) in the diagnosis of visceral leishmaniasis, by using the citrate-saline/formaldehyde (CSF) as an antigen diluent and anti-clumping/preservative agent instead of normal saline as originally instructed. Six FD-DAT antigen aliquots reconstituted with CSF volumes of $5,7,9,11,12$, or $13 \mathrm{~mL}$ with corresponding promastigote concentrations of $9.0 \times 10^{7}, 6.5 \times 10^{7}, 4.7 \times$ $10^{7}, 2.3 \times 10^{7}, 1.4 \times 10^{7}$, and $0.8 \times 10^{7} / \mathrm{mL}$ were tested against standard VL positive (+) and VL negative (-) sera starting at 1:100 serum dilution up to $1: 6,400$. Reading of the test results was similar for all six antigen aliquots by locating a circumscribed blue spot (end point) in the titration row that resembles the one in the control well containing sample diluent only (extreme left column); the serum dilution that preceded the end point is considered the titer of the serum sample. Titers $\geq 1: 3,200$ are indicative of VL. All six antigen aliquots showed similar test readings: titers $\geq 1: 6,400$ in the $V L(+)$ and $\leq 1: 100$ in the non-VL (-). The antigen aliquot reconstituted with the highest CSF volume $(13 \mathrm{~mL})$ had the corresponding lowest promastigote concentration $\left(0.8 \times 10^{7}\right)$ and showed invalid agglutination reaction. This figure appears in color at www.ajtmh.org. 
reconstitution $\left(9.0 \times 10^{7} / \mathrm{mL}\right)$, a $140 \%$ increase in antigen volume can be regained, thus achieving a reduction of $\pm 150 \%$ in the test application cost $(r=0.9711 ; P<0.001)$ (Table 2). Furthermore, by reviving the validity of the expired FD-DAT batches, large antigen quantities can be spared from disposal.

A further reduction in the FD-DAT application cost was achieved through substituting fetal bovine serum (FBS) by gelatin in the sample diluent. Besides stability for 5 years at $40^{\circ} \mathrm{C}$, gelatin is also much cheaper ( $\$ 0.002 /$ test) than FBS (\$0.04/test). Under the current conditions of frequent and long-duration electric failures in Sudan, FBS is furthermore readily perishable.

Performance of the antigen reconstituted with CSF at the minimal promastigote concentration determined $\left(1.4 \times 10^{7}\right.$ / $\mathrm{mL}$ ) proved to be satisfactory (Table 3 ). All $21 \mathrm{VL}$ genuinely infected cases scored titers significantly higher than the cutoff $(1: 3,200)$. Whereas in the 10 Croatian dogs with canine visceral leishmaniasis, titers ranged between $1: 6,400$ and 1 : 819,200 , much lower values $(\leq 1: 400)$ were recorded in all 11 dogs with non-VL conditions. Similarly, all the 22 healthy Sudanese police dogs tested showed the lowest titer range (Table 3). Highly concordant ( $\mathrm{Fr}=15.3167 ; P=0.0005)$ titer readings were further demonstrated in particular between the CSF-reconstituted antigen and its equivalent in the LQ-DAT (Table 4).

Stability of the CSF-reconstituted antigen in both the nonexpired "valid" and repaired "expired" FD-DAT batches was clearly reflected in its consistent reactivity throughout the storage period (360 days) when compared with the original (24-48 hours) using normal saline.

Execution of the CSF-modified FD-DAT on the single sample dilution basis, as performed here, proved equally reliable as the full-out titration procedure (Table 5). Significantly smaller antigen volumes $(100 \mu \mathrm{L})$ than in the full-out titration $(1,500 \mu \mathrm{L}-2,100 \mu \mathrm{L})$ were, therefore, used (Table 5).

The replacement of $\beta$-ME by urea proved to have no unfavorable effect on the test results in 30 blood samples from patients with or without VL. In the original FD-DAT and the LQDAT, all of the eight VL cases scored titers $\geq 1: 12,800$ and all of the 22 non-VL cases $\leq 1: 200$, the same as in the CSF-modified FD-DAT (Fr $=9.6500 ; P=0.0080)$.

TABLE 2

Volumes of citrate-saline/formaldehyde used as antigen diluent in a reference FD-DAT against the corresponding promastigote concentration per unit suspension medium, the number of patients under investigation, and the application cost involved

\begin{tabular}{cccc}
\hline $\begin{array}{c}\text { Anti-clumping/ } \\
\text { preservative volumes } \\
\text { used for FD-DAT antigen } \\
\text { reconstitution (mL) }\end{array}$ & $\begin{array}{c}\text { Promastigote } \\
\text { concentration/mL } \\
\text { suspension }\end{array}$ & $\begin{array}{c}\text { Antigen available } \\
\text { for testing number } \\
\text { of cases }\end{array}$ & $\begin{array}{c}\text { FD-DAT application } \\
\text { cost per vial }\end{array}$ \\
\hline 5 & $9.0 \times 10^{7}$ & 8 & $\$ 32.0$ \\
6 & $7.8 \times 10^{7}$ & 10 & $\$ 25.6$ \\
7 & $6.5 \times 10^{7}$ & 11 & $\$ 23.3$ \\
8 & $5.4 \times 10^{7}$ & 13 & $\$ 19.7$ \\
9 & $4.7 \times 10^{7}$ & 15 & $\$ 17.0$ \\
10 & $3.4 \times 10^{7}$ & 17 & $\$ 15.0$ \\
11 & $2.3 \times 10^{7}$ & 18 & $\$ 14.2$ \\
12 & $1.4 \times 10^{7}$ & 20 & $\$ 12.8$ \\
13 & $0.8 \times 10^{7}$ & $\begin{array}{c}\text { Invalid test } \\
\text { outcome }\end{array}$ & nd \\
& & &
\end{tabular}

FD-DAT = freeze-dried direct agglutination test.

${ }^{*} \mathrm{nd}=$ Not estimated because of invalid test outcome.
TABLE 3

Diagnostic performance of a reference freeze-dried direct agglutination test modified by incorporating citrate-saline/formaldehyde as an antigen diluent for the detection of $\mathrm{VL}$ in the human $(\mathrm{VL})$ and $\mathrm{CVL}$ hosts

\begin{tabular}{clrrrrrrrr}
\hline Origin of blood/serum sample & Final diagnosis & \multicolumn{7}{c}{$\begin{array}{l}\text { Titers expressed as the number of the } \\
\text { 2-fold serial dilution }\end{array} \leq 23456+78 \geq 9$} \\
\hline Human (48) & VL (21) & 0 & 0 & 0 & 0 & 0 & 0 & 0 & 21 \\
& Non-VL (27) & 9 & 1 & 14 & 3 & 0 & 0 & 0 & 0 \\
Canine (43) & CVL (10) & 0 & 0 & 0 & 0 & 0 & 2 & 2 & 6 \\
& Non-CVL (33) & 29 & 1 & 3 & 0 & 0 & 0 & 0 & 0
\end{tabular}

$\mathrm{CVL}=$ canine visceral leishmaniasis; $\mathrm{VL}=$ visceral leishmaniasis

* Titers are expressed as the number of 2-fold serial dilution starting from 1:100 (dilution 1) up to $1: 25,600$ (dilution 9 ).

† Cutoff titer for VL and CVL = 1:3,200 (dilution 6).

\section{DISCUSSION}

Following the successful development of the liquid version, efforts were made at the Royal Tropical Institute (Amsterdam the Netherlands) to improve the stability of the DAT in countries such as Sudan where ambient temperatures of $\pm 40^{\circ} \mathrm{C}$ are considered normal during the period from April to October. A commercialized freeze-dried version (FD-DAT) was accordingly developed that demonstrated stability at high temperatures $\left(56^{\circ} \mathrm{C}\right)$ throughout a storage period of 18 months. ${ }^{11}$ Highly encouraging results using this test version were reported later on in Sudan. ${ }^{12,13}$ Despite freezing at ultra-low temperatures $\left(-80^{\circ} \mathrm{C}\right.$ to $\left.-85^{\circ} \mathrm{C}\right)$ followed by dehydration under pressure, the freeze-dried promastigote antigen showed sensitivity and specificity for VL detection almost similar to the liquid equivalent. With stability at much lower temperatures $\left(\leq 45^{\circ} \mathrm{C}\right)$ than the aforementioned $\left(56^{\circ} \mathrm{C}\right)$, a second freezedried version was developed at the Institute for Tropical Medicine in Antwerp, Belgium. ${ }^{14}$

Following extended laboratory and field evaluation during the past 28 years, a remarkably lower temperature range $\left(5-35^{\circ} \mathrm{C}\right)$ is indicated for the storage of the Dutch FD-DAT kit version. Our experience with this test version under laboratory conditions in Sudan (temperatures at $23-26^{\circ} \mathrm{C}$ and at $40-45^{\circ} \mathrm{C}$, respectively) showed that refrigeration $\left(4-5^{\circ} \mathrm{C}\right)$ is the best option for maintaining its validity for 2 years (unpublished observation). It remains unclear, however, why the Dutch FD-DAT version can no longer maintain stability at temperatures $>35^{\circ} \mathrm{C}$ because this feature, among the other properties, is considered essential for its use in major endemic areas, including Sudan.

Other than the LQ-DAT application with a settling time of \pm 2 hours, a 5-hour delay is noticed for settling of the promastigote

TABLE 4

Performance of modified reference FD-DAT test in comparison with original and the LQ-DAT versions currently used for the detection of $\mathrm{VL}$

\begin{tabular}{|c|c|c|c|c|c|c|c|c|}
\hline $\begin{array}{l}\text { Direct agglutination } \\
\text { test version }^{\star}\end{array}$ & \multicolumn{8}{|c|}{$\begin{array}{l}\text { Titers expressed as the number of the } 2 \text {-fold } \\
\text { serial dilutiont: } \leq 23456 \neq 78 \geq 9\end{array}$} \\
\hline $\begin{array}{c}\text { Citrate-saline/formald } \\
\text { improved FD-DAT }\end{array}$ & 2 & 1 & 12 & 3 & 0 & 0 & 0 & 1 \\
\hline Cur & 10 & e & & 0 & & & & \\
\hline Current LQ-DAT & 1 & 2 & 11 & 4 & 0 & 0 & 0 & 12 \\
\hline
\end{tabular}

FD-DAT = freeze-dried direct agglutination test; LQ-DAT = liquid direct agglutination test; $\mathrm{VL}=$ visceral leishmaniasis.

* No statistical difference between outcomes of the three test versions was found $(\mathrm{Fr}=$ 15.3167; $P<0.0001$.

$\dagger$ Titers are expressed as the number of the 2 -fold serial dilution starting from 1:100 (dilution

1) up to dilution $1: 25,600$ (dilution 9 ).

$\ddagger$ Cutoff for $\mathrm{VL}=$ (dilution 6, 1:3,200). 
TABLE 5

Application cost of a modified FD-DAT based on single sample dilution testing in comparison with the full-out titration procedure currently in use for the detection of visceral leishmaniasis

\begin{tabular}{|c|c|c|c|c|c|c|c|}
\hline \multirow{3}{*}{$\begin{array}{l}\text { Sample } \\
\text { number }\end{array}$} & \multicolumn{7}{|c|}{ Direct agglutination execution procedure based on } \\
\hline & \multicolumn{3}{|c|}{$\begin{array}{l}\text { Single dilution } \\
\text { (cutoff }=1: 3,200 \text { ) }\end{array}$} & \multicolumn{3}{|c|}{$\begin{array}{l}\text { Full-out titration starting } \\
\text { at } 1: 100 \text { sample dilution }\end{array}$} & \multirow[b]{2}{*}{ End titer } \\
\hline & Antigen & Result & Cost & Antigen & Result & Cost & \\
\hline 1065 & $100 \mu \mathrm{L}$ & + & $\$ 5.4$ & $1,500 \mu \mathrm{L}$ & + & $\$ 81.00$ & $1: 819,200$ \\
\hline 1067 & $100 \mu \mathrm{L}$ & + & $\$ 5.4$ & $2,000 \mu \mathrm{L}$ & + & $\$ 108.00$ & $\begin{array}{c}1: \\
26214400\end{array}$ \\
\hline 1074 & $100 \mu \mathrm{L}$ & + & $\$ 5.4$ & $2,100 \mu \mathrm{L}$ & + & $\$ 113.4$ & $\begin{array}{c}1: \\
104857600\end{array}$ \\
\hline 1053 & $100 \mu \mathrm{L}$ & - & $\$ 5.4$ & $100 \mu \mathrm{L}$ & - & $\$ 5.4$ & $1: 100$ \\
\hline 1055 & $100 \mu \mathrm{L}$ & - & $\$ 5.4$ & $100 \mu \mathrm{L}$ & - & $\$ 5.4$ & $1: 100$ \\
\hline 1064 & $100 \mu \mathrm{L}$ & - & $\$ 5.4$ & $100 \mu \mathrm{L}$ & - & $\$ 5.4$ & $1: 100$ \\
\hline
\end{tabular}

antigen to the bottom of the V-shaped well when the current FD-DAT is used on non-VL sera. Most likely, free settling of the parasites is affected because of the physicochemical change(s) on the promastigote surface or in the suspension medium (possibly because of freeze-drying). To some extent, this was also reflected on the partial Coomassie Blue stain shedding and the subsequent blue background formation around the negative blue dot that indicate the end-point of the reaction. The use of CSF as an anti-clumping/preservative agent for the antigen reconstitution resulted in faster promastigote settling ( \pm 2 hours) and in the formation of a more compacter and sharper edged blue dot that clearly signals the reaction end-point (Figure 1). ${ }^{15,16}$ The failure of the 7 -year expired antigen to do the same in the $\mathrm{V}$-shaped well containing the diluent only or the negative non-VL control sample seems most likely to be the reason for expiry.

The appreciable extension in antigen shelf-life ( $\geq 1$ year) using CSF can be attributed to the long-term effect of this anticlumping/preservative. No measurable effect on the diagnostic reliability of the test was observed, which was also confirmed by the concordant FD-DAT and LQ-DAT results (Tables 2 and 3 ). Instead of being disposed of, the "expired" FD-DAT batches can, therefore, be used.

Another factor that contributes to the high FD-DAT application cost is the excessive use of promastigotes in the antigen suspension. In other words, in vitro cultivation to produce the huge number of promastigotes needed for antigen preparation requires large quantities of ingredients, where the FBS in particular is extremely expensive $( \pm \$ 450 / L)$. Following procedures essentially similar to the African trypanosomiasis macro-agglutination test (CATT), promastigote concentrations as high as $1.0 \times 10^{8}$ or even $2.0 \times 10^{8} / \mathrm{mL}$ were used for the production of both the liquid and freeze-dried DAT versions. ${ }^{11,15-17}$ Because the criterion for reading the test results in all DAT versions, including the FD-DAT, is exactly the opposite to that of CATT, by locating the negative reaction (blue dot) versus the positive (mat formation) as in the CATT, significantly lower promastigote concentrations can, thus, be used (Figure 1). In this study, a low concentration $\left(1.4 \times 10^{7}\right.$ promastigotes $/ \mathrm{mL}$ ) has resulted in an outcome identical to the original test version $\left(9.0 \times 10^{7}\right)$.

The very limited usability of the diluted antigen ( $\leq 48$ hours) in the original FD-DAT has also appreciably added to an increase in the test application cost. Through incorporating the CSF a significant extension in the shelf-life of the reconstituted antigen both in the "valid" and "expired" batches is achieved. Similarly to LQ-DAT, the CSF-modified antigen in FD-DAT can now cope with the adverse conditions in the Sudan. The diagnostic reliability of this modified FD-DAT version was further confirmed by the matching results of the other two DAT versions (Tables 2 and 3 ).

Irrespective of the DAT version used, the purpose of executing the test on the basis of full-out titration is to determine the end titer level in the blood/serum sample. Although not in all cases, the titer level may give an indication how severe $V L$ is in those identified as positives (titers $\geq 1: 3,200$ ). Regardless of the titer level, all patients with positive results receive a similar standard treatment dose per kg body weight with first-line anti-leishmanial. ${ }^{18}$ Because the FD-DAT procedure based on full-out titration (titers up to 1: 52428800 or higher) requires large antigen volumes $(\geq 2,100 \mu \mathrm{L}=\$ 113.00)$, adopting the alternative approach by testing at a single sample dilution of 1 : 3,200 as VL cutoff $(100 \mu \mathrm{L}=\$ 5.4)$ will contribute significantly to the improvement of the test feasibility (Table 4).

Notwithstanding all improvements introduced, we still believe that the current FD-DAT lacks the required safety for routine and mass application because of the $\beta-M E$ use in the test procedure. In addition to being extremely odorous, the continuous exposure to this toxicant can also lead to serious effects on the eyes, the respiratory, and central nervous systems. ${ }^{19}$ Damage to the aquatic life has also been reported because of drain spillage. ${ }^{20}$ Based on the success achieved earlier with LQ-DAT, a similar test procedure was followed with CSF-improved FD-DAT. ${ }^{4}$ By using a minor concentration $(0.3 \% \mathrm{w} / \mathrm{v})$ of the far less toxic urea instead of $\beta-\mathrm{ME}$, an equally efficient and even safer test procedure is established. This urea-improved FD-DAT version has demonstrated the reliability almost similar to that of LQ-DAT (Fr $=9.6500 ; P=$ 0.0080 ), thus meeting our objective to improve, besides feasibility and stability, also the safety for application of FD-DAT to confirm LQ-DAT (Table 6).

The success achieved in revitalizing the antigen in an expired FD-DAT or prolonging its shelf-life in a non-expired (valid) one was based on results obtained in test batches with the lot numbers indicated. Further experiments are required to confirm whether this approach is equally effective for other FD-DAT batches. It is also important to check the diagnostic

TABLE 6

Performance of urea-improved FD-DAT in comparison with an original, $\beta$-ME LQ-DAT version

\begin{tabular}{llclc}
\hline $\begin{array}{c}\text { Direct agglutination } \\
\text { test version }^{2}\end{array}$ & $\begin{array}{c}\text { Clinical } \\
\text { status }\end{array}$ & $\begin{array}{c}\text { Number of } \\
\text { sera tested }\end{array}$ & $\begin{array}{c}\text { Direct } \\
\text { agglutination } \\
\text { test result }\end{array}$ & Titer range \\
\hline Urea-improved & VL & 8 & Positive & $\geq 1: 25,600$ \\
FD-DAT & Non-VL & 11 & Negative & $1: 100$ \\
& & 11 & Negative & $\leq 1: 50$ \\
Non-expired & VL & 5 & Positive & $\geq 1: 25,600$ \\
FD-DAT with & & 3 & Positive & $1: 12,800$ \\
$\beta-M E$ & Non-VL & 1 & Negative & $1: 200$ \\
& & 21 & Negative & $1: 100$ \\
Non-expired & VL & 8 & Positive & $\geq 1: 25,600$ \\
LQ-DAT with & Non-VL & 1 & Negative & $1: 200$ \\
$\beta-M E^{*}$ & & 2 & Negative & $1: 100$ \\
& & 19 & Negative & $1: 50$ \\
\hline$\beta-M E=\beta$-mercaptoethanol; FD-DAT = freeze-dried direct agglutination test; LQ-DAT =
\end{tabular}
liquid direct agglutination test; $\mathrm{VL}=$ visceral leishmaniasis.

${ }^{\star}$ DAT versions with the same symbol $\left({ }^{\star}\right)$ are not statistically different $(\mathrm{Fr}=9.6500 ; P=$ 0.0080). 
reliability of the modified/repaired test batches against established procedures such as the original FD-DAT or LQDAT before dispatching them to hospitals or regional laboratories in the endemic areas.

Received October 8, 2019. Accepted for publication January 2, 2020.

Published online February 10, 2020.

Acknowledgments: We thank Sayda H. ElSafi, faculty of medicine, University of Khartoum, Khartoum, Sudan; Arend Kolk, van't Hoff Institute for Molecular Sciences, University of Amsterdam, Amsterdam, The Netherlands; and Ibrahim I. Ibrahim, W. Hassan, and Y. Mahamoud, Ahfad University for Women, Omdurman, for their cooperation for the execution of this study, their advices, and reading of the manuscript.

Authors' addresses: Abdallah el Harith, Yousif Awad, Abdelhafeiz Mahamoud, and Hussam Ali Osman, School of Pharmacy, Ahfad University for Women, Omdurman, Sudan, E-mails: abdallah. elharith@gmail.com, yousifahfad@gmail.com, mabdelhafeiz@gmail. com, and hussomco@gmail.com. Elfadil Abass, Department of Clinical Laboratory Science, College of Applied Medical Sciences, Imam Abdulrahman Bin Faisal University, Dammam, Saudi Arabia, E-mail: emabass@uod.edu.sa. Durria Mansour, School of Health Sciences, Ahfad University for Women, Omdurman, Sudan, E-mail: durriaelhussein@gmail.com. Claudia Moura de Melo and Rubens Riscala Madi, Program in Health and Environment, University of Tiradentes, Aracaju, Brazil, E-mails: claudiamouramelo@hotmail.com and rrmadi@gmail.com. Saul J. Semiao-Santos, Department of Medicine and Nursing, University of Tiradentes, Aracaju, Brazil, E-mail: saulix@gmail.com.

\section{REFERENCES}

1. Boelaert M, Bhattacharya S, Chappuis F, El Safi SH, Hailu A, Mondal D, Rijal S, Sundar S, Wasunna M, Peeling RW, 2007. Evaluation of rapid diagnostic tests: visceral leishmaniasis. Nat Rev Microbiol 5: S30-S39.

2. Osman HA, Mahamoud A, Abass E, Madi RR, Santos SJ, el Harith $A, 2016$. Local production of a liquid direct agglutination test as a sustainable measure for control of visceral leishmaniasis in Sudan. Am J Trop Med Hyg 94: 982-986.

3. Mahamoud A, Osman HA, Abass EM, Agib A, Madi RR, Santos SJ, el Harith A, 2018. Identification of an area predominantly endemic for childhood and adolescent visceral leishmaniasis in central Sudan. Acta Trop 178: 142-147.

4. Mahamoud A, Awad Y, Osman HA, Agib A, Madi RR, SemiaoSantos SJ, el Harith A, 2018. User and environment friendly direct agglutination test for diagnosis of visceral leishmaniasis: exclusion of formaldehyde and $\beta$-mercaptoethanol in test execution. J Med Microbiol 67: 1731-1736.

5. el Harith A, Mahamoud A, Awad Y, Mansour D, Abass E, Agib A, Madi RS, Semiao-Santos SJ, Osman HA, 2019. Are we well prepared for another major visceral leishmaniasis epidemic in Sudan? Open Forum Infect Dis 6: 1-7.

6. el Harith A, Kolk AHJ, Leeuwenburg J, Muigai R, Huigen E, Jelsma T, Kager PA, 1988. Improvement of a direct agglutination test for field studies of visceral leishmaniasis. J Clin Microbiol 26: 1321-1325.
7. el Harith A, Chowdhury S, Al-Masum A, Santos SS, Karim E, elSafi S, Haque I, 1995. Evaluation of cleaving agents other than trypsin in direct agglutination test for further improving diagnosis of visceral leishmaniasis. J Clin Microbiol 33: 1984-1988.

8. el Harith A, Kolk AHJ, Kager PA, Leeuwenburg J, Faber FJ, Muigai R, Kiugu S, Laarman JJ, 1987. Evaluation of a newly developed direct agglutination test (DAT) for sero-diagnosis and seroepidemiological studies of visceral leishmaniasis: comparison with IFAT and ELISA. Trans R Soc Trop Med Hyg 81: 603-606.

9. el Harith A, el Mutasim M, Mansour D, Mustafa F, Ervidson H, 2003. Use of glycerol as alternative to freeze-drying for longterm preservation of antigen for the direct agglutination test. Trop Med Int Health 8: 1025-1029.

10. el Harith A, Kolk AHJ, Kager PA, Leeuwenburg J, Muigai R, Laarman J, 1986. A simple and economical direct agglutination test for the serodiagnosis and sero-epidemiological studies of visceral leishmaniasis. Trans R Soc Trop Med Hyg 80:583-587.

11. Meredith SE, Kroon NC, Sondorp E, Seaman J, Goris MG, van Ingen CW, Oosting H, Schoone G, Terpstra WJ, Oskam L, 1995. Leish-KIT, a stable direct agglutination test based on freezedried antigen for serodiagnosis of visceral leishmaniasis. J Clin Microbol 33: 1742-1745.

12. Zijlstra EE, Osman OF, Hofland HW, Oskam L, Ghalib HW, elHassan AM, Kager PA, Meredith SE, 1997. The direct agglutination test for diagnosis of visceral leishmaniasis under field conditions in Sudan: comparison of aqueous and freeze-dried antigens. Trans $R$ Soc Trop Med Hyg 91: 671-673.

13. Abdallah KA, Nour BY, Schallig HD, Mergani A, Hamid Z, Elkarim AA, Saeed OK, Mohamadani AA, 2004. Evaluation of the direct agglutination test based on freeze-dried Leishmania donovani promastigotes for the serodiagnosis of visceral leishmaniasis in Sudanese patients. Trop Med Int Health 9: 1127-1131.

14. Jacquet D, Boelaert M, Seaman J, Rijal S, Sundar S, Menten J, Magnus E, 2006. Comparative evaluation of freeze-dried and liquid antigens in the direct agglutination test for serodiagnosis of visceral leishmaniasis (ITMA-DAT/VL). Trop Med Int Health 11: $1777-1784$.

15. Schoone GJ, Hailu A, Kroon CCM, Nieuwenhuys JL, Schallig HDFH, Oskam L, 2001. A fast agglutination screening test (FAST) for the detection of anti-Leishmania antibodies. Trans $R$ Soc Trop Med Hyg 95: 400-401.

16. Hailu A, Kroon CC, Schoone GJ, Berhe N, Schallig HD, Kager PA, 2002. sero-epidemiological assessment and diagnosis of visceral leishmaniasis in an endemic locality using fast agglutination screening test (FAST). Acta Trop 83: 93-101.

17. Magnus E, Vervoort T, van Miervenne N, 1978. A card agglutination test with stained trypanosomes (C.A.T.T.) for the serological diagnosis of T.B. gambiense trypanosomiasis. Ann Soc Belg Med Trop 58: 169-176.

18. de Beer P, el Harith A, Deng LL, Semiao-Santos SJ, Chantal B, van Grootheest, 1991. A killing disease epidemic among displaced Sudanese population identified as visceral leishmaniasis. Am J Trop Med Hyg 44: 283-289.

19. White K, Bruckner JV, Guess WL, 1973. Toxological studies of 2mercaptoethanol. J Pharm Sci 62: 237-241.

20. de Jonge M, Teuchies J, Meire P, Blust R, Bervoets L, 2012. The impact of increased oxygen conditions on metal-contaminated sediments part II: effects on metal accumulation and toxicity in aquatic invertebrates. Water Res 46: 3387-3397. 